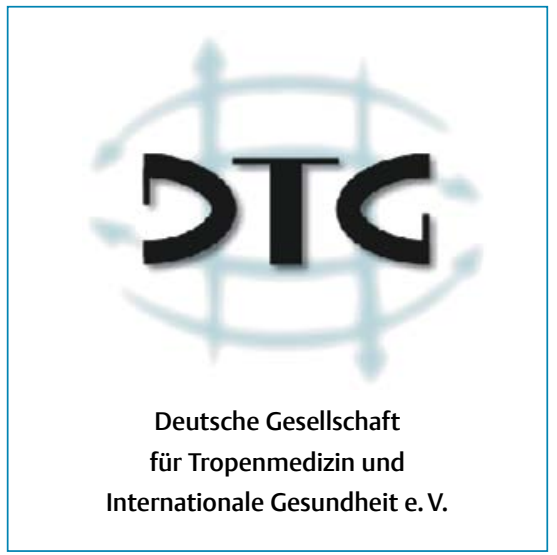

\title{
Liebe DTG-Mitglieder und Leser der FTR,
}

nach Rückkehr aus dem Einsatz in Afghanistan, der mich mit einigen, auch tropenmedizinischen Aspekten in diesem heißen, trockenen und harten Land konfrontiert hat, lese ich von dem unglücklichen USSoldaten, der zwar den ISAF-Kampfeinsatz heil überstanden hatte, dann aber 2 Monate nach seiner Heimkehr an Rabies gestorben ist. Was für ein vermeidbarer und unsinniger Tod 7 Monate nach dem Biss durch einen streunenden Hund im afghanischen Camp. Entsprechend fällt die Reaktion seiner Familie aus, die mittels Telefon die Entwicklung des Krankheitsbilds Schritt für Schritt mitverfolgen musste. Ganz offenbar liegt eine unselige Kombination aus fehlender Präexpositionsprophylaxe und schlampiger postexpositioneller Impfung vor. Weitere Details sind bisher offiziell nicht mitgeteilt worden. Die Untersuchung des Streuners habe ein negatives Ergebnis erbracht. Man fragt sich, wo und wie diese Diagnostik erfolgt ist.

Während meines Einsatzes hatte ich auch Gelegenheit Health Posts in kleineren Dörfern in den Bergen in der Umge-
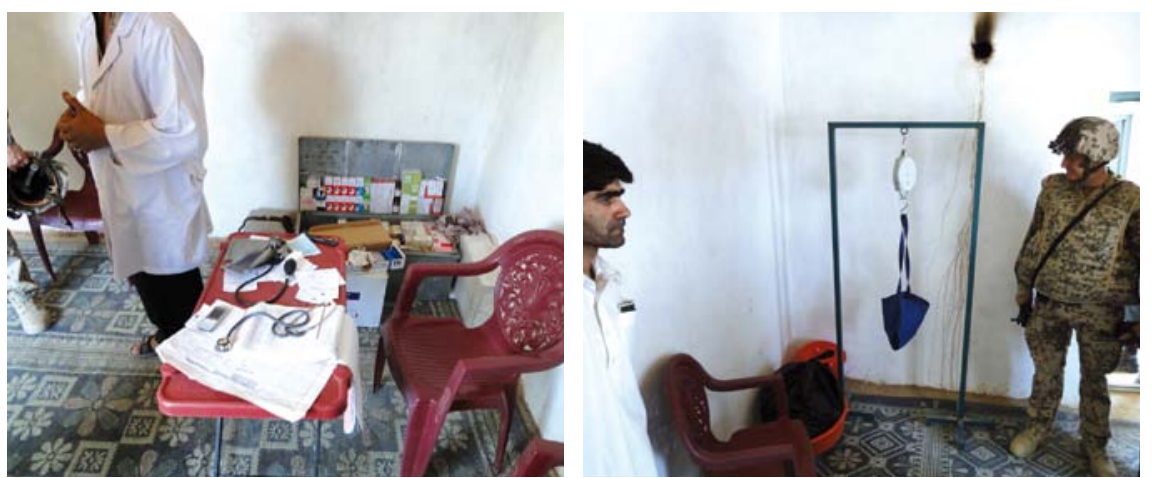

Abb. 1 und 2 Medizinische Versorgung durch engagierte Pflegekräfte und Ärzte in Health Posts in der Umgebung von Kunduz, Afghanistan.

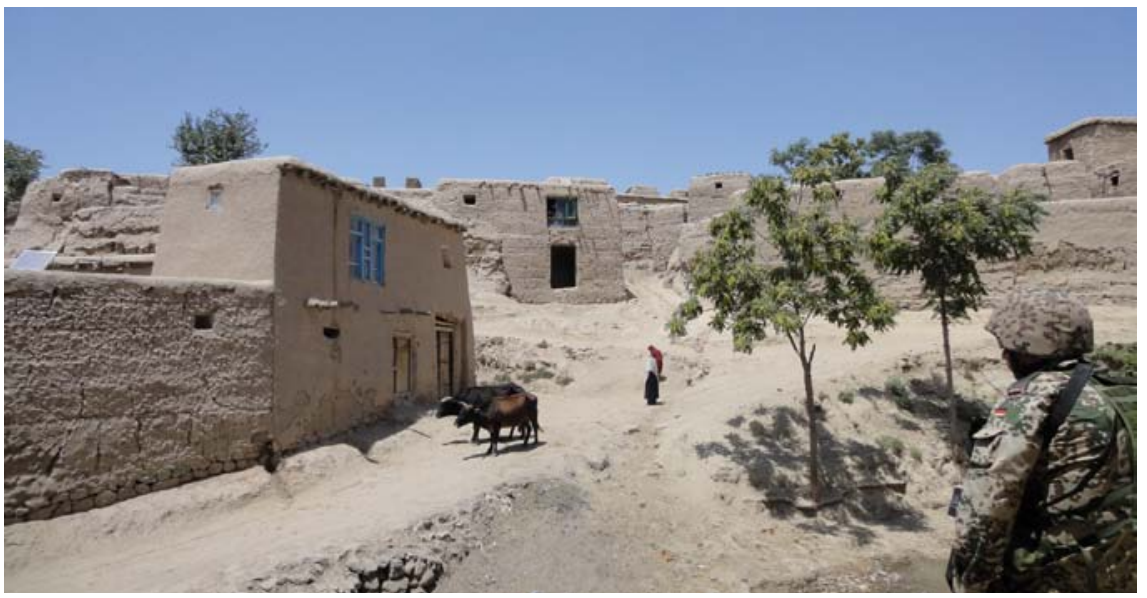

Abb. 3 Schwierige infrastrukturelle und klimatische Bedingungen für eine Grundversorgung. bung von Kunduz zu besuchen. Dort halten einige engagierte Pflegekräfte und Ärzte eine medizinische Grundversorgung unter schwierigen klimatischen und infrastrukturellen Bedingungen aufrecht. Die private Hilfsorganisation wird vom Auswärtigen Amt beziehungsweise dem Ministerium für wirtschaftliche Zusammenarbeit finanziert und betreibt auch in der Stadt Taloquan eine funktionierende Einheit für mangelernährte Kleinkinder sowie eine $\mathrm{HNO}$ - und $\mathrm{Au}-$ genklinik.

Krankheiten aus dem Umfeld der Tropenmedizin erleben wir jedoch auch immer wieder vor unserer Haustür. In beziehungsweise in der Nähe meines Urlaubsorts in Südwestfrankreich gab es in den letzten Jahren mehrere Vorfälle: Erst mussten nach dem Auftreten von Tollwut alle Hunde eines Departments in einer teuren und aufwendigen Aktion geimpft werden. Dann haben sich 2 Menschen an der Riviera mit Arboviren infiziert. Dieses Jahr erkrankten mehrere Rinder der Rasse Blondes de Aquitaine an Rindertuber- 
halb notgeschlachtet.

Beiträge in FTR und auf www.dtg.org Wir wollen Sie ermuntern, Ihre Erfahrungen, Anregungen und Aktivitäten auf diesen Seiten zu veröffentlichen und damit an andere Mitglieder weiterzugeben. Das kann ein Text oder auch die Abbildung eines interessanten Befunds oder einer typischen Situation aus den Tropen sein. Wenn Sie diese Zeilen lesen, ist der Europäische Kongress in Barcelona bereits vorüber und wir werden hoffentlich in dem einen oder anderen Beitrag darüber berichten können. Nicht jeder hat ja die Zeit und Gelegenheit nach Barcelona zu fahren.

Jahreshauptversammlung März 2012

Wir bitten Sie auch um Ihre Vorschläge und Ihre Unterstützung für die Vorbereitungen unserer Versammlung im März 2012. Die Vorschlagsfrist für Vorstandsmitglieder ist satzungsgemäß am 1. Oktober abgelaufen. Im Rahmen der Versammlung werden wir jedoch auch den Preis für Tropenmedizin 2012 an einen jungen Wissenschaftler vergeben. Vorschläge und Bewerbungen können bis Ende des Jahres eingereicht werden. Alle weiteren Informationen sind dazu auf der Homepage, http://www.dtg.org/91.html.

Veranstaltung für jüngere Mitglieder Eine Veranstaltung gerade für die jüngeren Mitglieder der DTG, für Studierende und alle, die in den Tropen arbeiten und lernen wollen, findet am 12.11.2011 von 10 bis 18 Uhr, Hörsaal im Haus Michael, Mariannhillstr. 1c, 97074 Würzburg, veranstaltet vom Forum für Internationale Gesundheit, www.foring.org, statt.

Es grüßen Sie herzlich

Prof. Dr. Thomas Löscher und Dr. Hinrich Sudeck

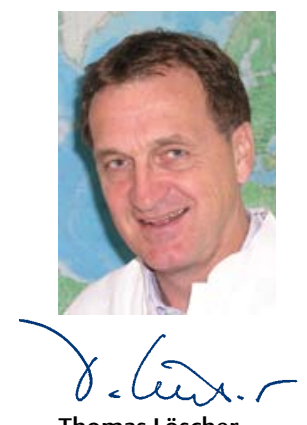
München
Thomas Löscher,

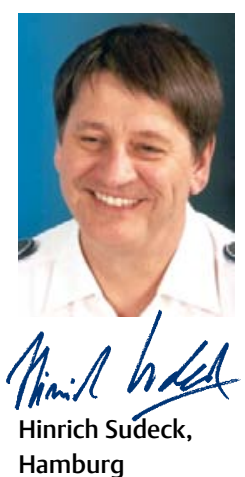

Ankündigung

\section{DTG-Jahrestagung 2012}

Die nächste Jahrestagung der DTG wird vom 14.-17.03.2012 in Heidelberg als gemeinsame Tagung mit der Deutschen Gesellschaft für Parasitologie stattfinden. Die Tagung wird für die DTG von Prof. Dr. Thomas Junghanss und seinem Team organisiert. Prof. Junghanss und der DTGVorstand laden Sie herzlich zu dieser DTG/DGP-Tagung nach Heidelberg ein. Ein besonders attraktives Programm mit einem breiten Themenspektrum ist in

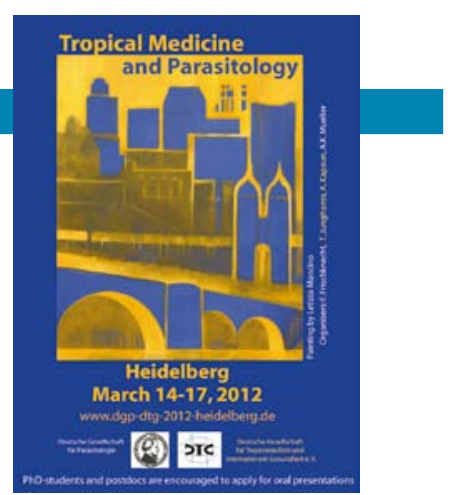

Vorbereitung. Anregungen und Vorschläge zu Symposien und Workshops sind willkommen. Details zur Anmeldung und zur Einreichung von Abstracts finden Sie auf der DTG-Homepage, www.dtg.org.

\section{Aktualisierung Leitlinie}

\section{Diagnostik und Therapie der Malaria}

Die S1-Leitlinie zur Diagnostik und Therapie der Malaria wurde vom DTG-Ausschuss ,Leitlinien“ unter der Federführung von Prof. Dr. Gerd-Dieter Burchard aktualisiert und ist seit dem 23. August auf der Homepage sowohl der DTG, www. dtg.org, wie auch der AWMF, www.awmf. org, abrufbar.

Weitere DTG-Leitlinien gibt es zur

- Diagnostik und Therapie der Amöbenruhr
- Diagnostik und Therapie des Amöbenleberabszesses

- Diagnostik und Therapie der viszeralen Leishmaniasis (Kala-Azar)

- Diagnostik und Therapie der kutanen und mukokutanen Leishmaniasis

- Diagnostik und Therapie der Schistosomiasis (Bilharziose)

Alle Leitlinien sind auf der DTG-Homepage abrufbar.

\section{Ausschreibung}

\section{Preis für Tropenmedizin 2012}

Die Deutsche Gesellschaft für Tropenmedizin und Internationale Gesundheit (DTG e. V.) schreibt auch dieses Jahr einen Preis für Tropenmedizin zur Förderung jüngerer Wisse nschaftler aus. Der Preis wird für besondere wissenschaftliche Leistungen auf dem Gebiet der Tropenmedizin und der internationalen Gesundheitswissenschaften verliehen und ist mit 2500 Euro dotiert. Bewerbungen und Vorschläge richten Sie bitte bis zum 31.12.2011 an den Schriftführer der DTG:

Dr. Hinrich Sudeck

Bundeswehrkrankenhaus Hamburg, Fachbereich Tropenmedizin

Bernhard-Nocht-Straße 74, 20359 Hamburg

Einzureichen sind in 3-facher Ausfertigung:

- Lebenslauf,

- Liste der Publikationen,

- die für die Bewerbung wesentlichen Veröffentlichungen oder Manuskripte.

- Zusammenfassung der wissenschaftlichen Leistungen (maximal eine DIN-A4Seite), aufgrund derer die Bewerbung eingereicht wird.

Die Bewerber sollten nicht älter als 40 Jahre alt sein. Die Entscheidung des Vorstands der Gesellschaft über die Auswahl des Preisträgers ist endgültig. Der Rechtsweg bleibt ausgeschlossen.

Die Preisverleihung findet im Rahmen der Jahrestagung der DTG in Heidelberg vom 14.-17. März 2012 statt. Informationen finden Sie auch auf der DTG-Homepage, http:// www.dtg.org/91.html. 